\title{
Editorial
}

Louisa $\mathrm{Ha}$

\section{Online Media and Global Communication: A Vision to be an Innovative Global Academic Publishing Model and an Olympic Game of Communication Scholars}

https://doi.org/10.1515/omgc-2022-0011

Do we really need another communication journal with so many new journals out there? Yes, at least for our journal, Online Media and Global Communication (OMGC). It is because although we have hundreds of communication journals and 94 are indexed by the Web of Science in 2021 (https://www.bgsu.edu/arts-and-sciences/media-andcommunication/resources/ssci-communication-journal-publishing-guide), but none are truly global, at least in the language they use. They are limited by either using only the English language, as the lingua franca of the academe, or the native language of the country the journal publishes. A few journals use a bilingual format such as English and Spanish, but none really encompass the official six languages of the United Nations which cover billions of people in the world: Arabic, Chinese, English, French, Russian, and Spanish. OMGC attempts to break through this language barrier by offering abstracts in these six languages and in a structured format so that readers in non-English speaking countries can have immediate access and understanding of the research we publish and can translate it further with the many translation engines available online. We hope to offer an innovative global academic publishing model.

The Winter Olympics is going to be held in China 2022. This reminded us that scholars, same as athletes, compete for excellence in a venue that is open to all countries. A good host is important and OMGC aspires to be that good host and venue for scholars around the globe to share their research, new theories, and methods on online media and global communication. We embrace a free open access model to both authors and readers with the generous sponsorship of the School of Journalism and Communication and the Center for Global of Public Opinion of China of the Shanghai International Studies University in China. The university not only provides funding and staffing, but also a rich resource of

Louisa Ha, Founding-Editor-in-Chief, Professor of Research Excellence, Bowling Green State University, Bowling Green, USA, E-mail: louisah@bgsu.edu 
translation talents for the journal. Our publisher, De Gruyter, is a German academic publisher with 273 years of history. Our editorial team and editorial board members include 22 men and 15 women, renowned scholars from 26 countries (see Editorial section on our journal web site: https://www.degruyter.com/omgc).

There are two websites for the journal. The official journal web site (https:// www.degruyter.com/omgc), hosted by De Gruyter, only focuses on articles and metrics about the journal. Our Shanghai International Studies University journal web site (http://omgc.shisu.edu.cn/12332/list.htm) contains news about the journal, events that our journal organized such as the upcoming symposium on the Misinformation and Global Communication on March 12, 2022 and other global communication research resources for scholars worldwide.

The rise of global online media, such as social media, blogs, mobile apps, podcasts, messaging apps, video streaming sites, etc., calls for more understanding on how these global media affect the world, especially in the field of global communication. Research in global online media is growing with the aid of computational, big data techniques. Researchers now can easily collect and analyze large amount of online media data in their own countries and other countries through webscrapers, Python computer programming, and machine learning. Tools such as online survey software and cross-national panels also facilitate research across countries.

OMGC aims to publish high quality, innovative, and original research on global communication especially in the use of global online media platforms such as Facebook, TikTok, YouTube, Twitter, Instagram, WhatsApp, Weibo, WeChat, Wikipedia, web sites, blogs, etc. We hope to make theoretical contributions based on practices of online media and global communication, particularly in the Global South. This journal will address the contemporary concerns about the effects and operations of global digital media platforms on international relations, international public opinion, fake news and propaganda dissemination, social change, consumer behavior as well as the balance of voices in the world. Meanwhile, the journal will address issues of global communication as related to the development of Smart Cities around the world, a UNESCO proposed concept, as global cities will constitute a dominating "media” platform for global communication in the future.

Studies that examine significant topics on theories and methods in global communication discourses between countries in both offline and online media or comparison of online and non-online media are within the scope of the journal. Comparative research studies across countries are particularly welcome. Single country studies that focus on online media with international implications will also be considered. Empirical research is preferred over conceptual papers. Descriptive studies with a nationally representative sample that can serve as benchmarks for a topic will also be considered. 
In addition to original research articles, OMGC will feature one invited research trend and state-of-the-art review essay in each issue on the online media and global communication research in a non-English speaking country or region. In addition, one highly commended translated article published in non-English native language communication journals as "Gem from the Global South" pertinent to the journal's scope will be added to each issue for readers' reference.

This journal adopts a dual track review system as the first in the field of communication to the best of my knowledge. Two review tracks are offered to the authors and authors must choose either one before final submission: 1) doubleblind (masked) review track that both authors and reviewers do not know the identity of the author; 2) single-blind (masked) review track that reviewers will know the identity of the author and the authors are free to cite their own works and refer to themselves in the manuscript. Submitters who have posted their manuscript or its earlier version elsewhere online or with more than five self-citations will undergo the review process using the single-blind review track. This system will maximize the number of possible highly qualified reviewers and allow authors with different situations to select the most appropriate route for reviewing their manuscripts. For the benefits and reasons for a dual review system, see my blog post in Peer Review Week (https://blog.degruyter.com/fact-or-fake-the-questionof-anonymity-in-peer-review/) and editorial essay published in Journalism and Mass Communication Quarterly (https://journals.sagepub.com/doi/full/10.1177/ 1077699020904383).

To create a fair citation environment for cultures that use full names with few last name differentiations and those cultures that differentiate mainly by last names, we use our house citation style which is highly similar to APA style but require full name/first name listing in the reference section. We welcome manuscripts in all citation styles in initial submissions. After all, the content of the paper is the most important determinant of its value to publish. For submission guideline to the journal, please visit our journal web site. We use the ScholarOne submission platform for manuscript submission (https://mc.manuscriptcentral.com/omgc).

\section{Highlights of Our Articles}

In this inaugural issue, we have four excellent research articles that demonstrate how our journal works and reflect the diversity of our article types. Two are singleblind refereed articles and two are double-blind refereed articles. The first article is Jörg Matthes' "Social Media and the Political Engagement of Young Adults: Between Mobilization and Distraction.” This article used longitudinal aggregate data and compared voter turnout and social media use data of young adults in U.S., 
Germany, Switzerland, and Japan to show how social media use can significantly dampen political engagement: Most young adults use social media primarily for non-political purposes, which distracts rather than mobilizes political participation.

Shahira Fahmy, Basma Mostafa Taha, and Hasan Karademir's article, “Journalistic Practices on Twitter: A Comparative Visual Study on the Personalization of Conflict Reporting on Social Media," shows how journalists working for news media in Iran, Qatar, Saudi Arabia, and the U.S. personalized their reporting of the controversial Yemen Civil War by examining the patterns of visual framing on their own Twitter accounts. It further explores the influence of the individual level factor (home country or foreign identity of the journalist) and organizational level factor (countries affiliated with news organizations directly or indirectly involved in the conflict), on images shared on the Twitter platform. They found that while journalists offered some personalized reporting, they preferred to mostly adopt a neutral stance when reporting the conflict.

Sherwin Chua and Oscar Westlund's article, "Platform Configuration: A Longitudinal Study and Conceptualization of a Legacy News Publisher's Platform-related Innovation Practices," is a longitudinal qualitative case study of a Singaporean legacy news publisher on how publishers are innovating their practices in the face of global platform companies' growing dominance on journalism. They propose platform configuration as a conceptual framework in analyzing the innovation in the six stages of news production.

Xuechen $\mathrm{Hu}$, Xingjian Gao, Wenzhen $\mathrm{Xu}$, and Jiro Takai's article, "Media Exposure and Risk Perception as Predictors of Engagement in COVID-19 Preventive Behaviors: Extending the Theory of Planned Behavior Across Two Cultures," compared the psychological and social factors that affect the performance of preventive behaviors toward COVID-19 by testing a model based on the Theory of Planned Behavior (TPB) on U.S. and Japanese people. They found that social networking site (SNS) involvement only affects Japanese but not U.S. people's attitudes toward COVID-19.

Our invited refereed review article is by Yong $\mathrm{Hu}$ and Lei Chen on Internetbased communication research published in four core communication journals in China. It traces the growth of the research since 1994 and the achievements in five key areas, reflects on the failures, and calls for a return to the study of fundamental relationship between communication and people in online communication.

To conclude this inaugural issue is our Gem from the Global South. We translated the article by Thaiane Oliveira published in 2020 originally in Portuguese in the Brazilian journal, Revista Fronteiras - estudos midiáticos, with author and journal permission. Titled, "Scientific Disinformation in times of Epistemic Crisis: Circulation of Conspiracy Theories on Social Media Platforms," it is also 
related to COVID-19 but focuses on the how conspiracy theories circulated on Whatapps, Facebook, and YouTube in Brazil using network analysis and participant observation. She shows that even though there is distrust about the relationship between science, government and industry, scientific authority is still a symbolic capital of extreme importance for the circulation of information on conspiracy theories related to science.

Happy Reading! 